\title{
Brain Asymmetry Detection and Machine Learning Classification for Diagnosis of Early Dementia ${ }^{\dagger}$
}

\author{
Nitsa J. Herzog ${ }^{1}\left(\mathbb{D}\right.$ and George D. Magoulas ${ }^{1,2, * \mathbb{C}}$
}

1 Department of Computer Science, Birkbeck College, University of London, London WC1E 7HZ, UK; nitsa@dcs.bbk.ac.uk

2 Birkbeck Knowledge Lab, University of London, London WC1E 7HZ, UK

* Correspondence: gmagoulas@dcs.bbk.ac.uk

+ Data used in preparation of this article were obtained from the Alzheimer's Disease Neuroimaging Initiative (ADNI) database (adni.loni.usc.edu). As such, the investigators within the ADNI contributed to the design and implementation of ADNI and/or provided data but did not participate in analysis or writing of this report. A complete listing of ADNI investigators can be found at: http://adni.loni.usc.edu/wp-content/uploads/how_to_apply/ADNI_Acknowledgement_List.pdf.

check for updates

Citation: Herzog, N.J.; Magoulas, G.D. Brain Asymmetry Detection and Machine Learning Classification for Diagnosis of Early Dementia . Sensors 2021, 21, 778. https://doi.org/ $10.3390 /$ s21030778

Received: 27 December 2020

Accepted: 21 January 2021

Published: 24 January 2021

Publisher's Note: MDPI stays neutral with regard to jurisdictional claims in published maps and institutional affiliations.

\begin{abstract}
Early identification of degenerative processes in the human brain is considered essential for providing proper care and treatment. This may involve detecting structural and functional cerebral changes such as changes in the degree of asymmetry between the left and right hemispheres. Changes can be detected by computational algorithms and used for the early diagnosis of dementia and its stages (amnestic early mild cognitive impairment (EMCI), Alzheimer's Disease (AD)), and can help to monitor the progress of the disease. In this vein, the paper proposes a data processing pipeline that can be implemented on commodity hardware. It uses features of brain asymmetries, extracted from MRI of the Alzheimer's Disease Neuroimaging Initiative (ADNI) database, for the analysis of structural changes, and machine learning classification of the pathology. The experiments provide promising results, distinguishing between subjects with normal cognition (NC) and patients with early or progressive dementia. Supervised machine learning algorithms and convolutional neural networks tested are reaching an accuracy of $92.5 \%$ and $75.0 \%$ for NC vs. EMCI, and $93.0 \%$ and $90.5 \%$ for NC vs. AD, respectively. The proposed pipeline offers a promising low-cost alternative for the classification of dementia and can be potentially useful to other brain degenerative disorders that are accompanied by changes in the brain asymmetries.
\end{abstract}

Keywords: asymmetry detection; brain asymmetry; brain MRI; dementia; machine learning methods; SVM; deep learning

\section{Introduction}

Dementia is a brain disorder that affects normal brain function due to the loss of neurons or neurons' functionality. Dementia may include a group of symptoms such as memory loss, lack of reasoning and judgment, problems with speech and understanding language, and changes in personality [1]. A total of 46.8 million people worldwide have dementia, and approximately 9.9 million new cases are registered every year. The proportion of dementia in the general population aged 60 and over is $7.1 \%$.

There are several types of degenerative diseases accompanied by dementia. Alzheimer's disease (AD) is the most common one, followed by vascular dementia, Lewy body dementia, Frontotemporal dementia, Parkinson's disease, and Huntington's disease.

Early dementia or amnestic Mild Cognitive Impairment (aMCI) is characterized by minor problems with memory, speech, or decision-making. More than $80 \%$ of people satisfying the definition of aMCI progress to Alzheimer's disease within 6 years. Early detection and identification of the structural and functional cerebral changes are crucial for providing proper care and treatment. Computer-aided diagnosis (CAD) of the anatomical changes 
in the brain, based on magnetic resonance imaging (MRI), gives accurate results for early diagnosis of brain disorders [2,3].

The current study is based on the hypothesis that brain asymmetry changes as a result of the development of early and progressive dementia. The evaluation of asymmetries in the cortex of the brain is based on structural Magnetic Resonance Imaging (sMRI). This research aims to investigate the pattern of these changes using MRI and computer vision techniques. The paper proposes an algorithm for segmenting and visualizing the differences in the symmetry between right and left hemispheres of the brain and generating asymmetry features. This is mainly focused on the early changes in the brain cortex when the clinical diagnosis is not obvious and cannot be done by medical professionals using traditional diagnostic methods. The pattern of brain asymmetries in a group of patients with normal cognition (NC), mild cognitive impairment (MCI) and dementia, very mild stable, and progressive to Alzheimer's Disease (AD) is analyzed with the help of statistical features. The information collected from image asymmetries can be sent for further processing and classification. The paper verifies the robustness of the generated brain asymmetry images and features, demonstrating their potential to produce consistent performance across different classification models. The rest of the article is organized as follows. Section 2 describes the problem and provides a review of the area. Section 3 introduces the Alzheimer's Disease Neuroimaging Initiative (ADNI) database used in this study and derives the data processing pipeline, including the approach for detection and analysis of image asymmetries and the machine learning methods used for classification. Results are presented in Section 4. The paper ends with a general discussion of the area and directions for future research in the field of brain asymmetries.

\section{Background of the Study}

The investigation of the anatomical properties and functional ability of the human brain is an intensively developing research area. The human brain has an overall leftward posterior and rightward anterior asymmetry (known as Petalia and Yakovlevian torque) [4]. The right cerebral hemisphere protrudes forward, and the left hemisphere protrudes backward compared to the right one. This type of asymmetry is mostly found in righthanded individuals — around $90 \%$ of the human population is right-handed [5] — while the opposite pattern is observed in left-handed individuals. The brain asymmetry is associated with lateralization that is a structural and functional difference between the left and right sides of the brain. These asymmetries originate from genetic and epigenetic factors in the evolutionary development of the brain [6,7]. The brain structure is more lateralized in males than in females [8]. It also depends on the age of the person. The brain activity in the frontal lobes of young adults is more lateralized than in elderly healthy individuals, whose brain becomes more symmetrical with binarized activities in both hemispheres $[9,10]$. The degree of structural asymmetry is correlated with the degree of functional lateralization. The left hemisphere is mostly responsible for language processing and logical thinking. For example, it includes the Broca's speech area and the Wernicke's language comprehension area. The right hemisphere specializes in musical and artistic abilities, spatial recognition, attention, and emotions [7].

The analysis of brain symmetry helps in the diagnosis of brain-related disorders [11]. The brain regions show a progressive decrease in the degree of asymmetry in patients with Mild Cognitive Impairment (MCI) and an increase in asymmetry in patients with Alzheimer's disease (AD) [12]. Left-hemisphere regions are affected earlier and more severely. The right cerebral regions become dominant in AD patients, but not in the early phase of the MCI. The abnormal hemispheric asymmetries of $\mathrm{AD}$ and $\mathrm{MCI}$ patients significantly correlate with functional brain activities and memory performance. Patients with MCI show an increase in the activation of many brain regions in the right hemisphere during the processing of word memory tasks [13]. Those areas are compensatorily activated compared to the activation zones in the left hemisphere of the healthy controls. The degree of asymmetry is not the same in the different parts of the brain [14]. Progressive demen- 
tia in patients with Alzheimer's disease is associated with a significant increase in the neuroanatomical asymmetries in subcortical brain structures such as the hippocampus and amygdala $[15,16]$. The research findings prove that shape analysis can detect the progression of dementia earlier than volumetric measures. Shape asymmetry, based on longitudinal asymmetry measures in the hippocampus, amygdala, caudate, and cortex can be a powerful imaging biomarker for the early presymptomatic prediction of dementia.

As with other types of imaging data processing, segmentation techniques are a necessity for detailed study of the anatomical regions of the brain and their symmetries. Brain segmentation is an important task in brain analysis and often the most critical step in many medical applications. Nowadays, many segmentation techniques offer the detection and separation of the whole brain tissues from the skull (skull stripping) or partial separation of the specific regions (gray or white matter) and individual brain structures in accordance with the anatomical atlas [17]. The current study proposes an algorithm for segmentation of the hemispheric asymmetries. The segmented areas usually require additional analytical tools for understanding their property in depth. Feature engineering gives knowledge about the most remarkable characteristics of the image [18]. Features detection and description refers to the procedure of identifying points of interest in an image (or object) that can be used to describe and analyze the image (object) contents [19], providing valuable data for image analysis. The current project includes an analysis part that is based on the evaluation of the statistical properties of imaging data. Statistical description of the image texture can generate a number of relevant and distinguishable features, which is crucial for the interpretation of the research findings.

Several studies in the machine learning area have highlighted the importance of feature selection for the improvement of classification performance [20,21]. For example, an effective feature selection method based on computing the chi-square statistical value was introduced in [22]. The diagnostic system of heart disease based on feature fusion, feature selection, and weighting techniques gives a high prediction of 98.5\% [23]. Zhou et al. [24] applied the C4.5 statistical (decision tree) classifier to select those weights from the gray matter (GM) regions that were most affected by the atrophic process. Using feature selection, the researchers were able to improve the classification performance of the proposed Transfer AdaBoost algorithm and achieved a classification accuracy of $85.4 \%$ for the ADNI database and 93.7\% for a local hospital dataset in the diagnosis of AD and MCI. $t$-test score ranking of the extracted features was proposed by Beheshti et al. [21] in a research work about discriminating between stable and progressive MCI cases. A genetic algorithm, equipped with the Fisher criterion function [25], evaluated the separation between the two groups of data and helped to select the most discriminative feature subsets for the classification with linear Support Vector Machine (SVM). The feature selection process raised the accuracy of the classification by $16 \%$. The average calculated accuracy reached $93.01 \%$ for stable $\mathrm{MCI}$ and $75 \%$ for progressive MCI. Another feature engineering technique combined with a Regularized Extreme Learning Machine (RELM) algorithm was proposed by Lama et al. [26]. The high-level PCA features [27] were chosen using the softmax function (a function that takes a vector of real numbers as input and normalizes it into a probability distribution). The proposed method showed $75.33 \%$ of accuracy for AD and $80.32 \%$ for MCI with binary classification and $76.61 \%$ with multiclass classification. Glozman and Le [28] developed machine learning methods, based on the architecture of white matter tracts, to classify Alzheimer's and healthy subjects. The ranking process was based on the differences in the average population values for each feature, whilst feature dimensionality was reduced using PCA. The researchers reported an increase in performance up to $92 \%$ after applying feature normalization and ranking.

Another popular method for the improvement of classification performance is the implementation of classifier ensembles [29]. They combine multiple meta-algorithms in one predictive model in order to minimize error and enhance predictive accuracy. Moradi et al. [30] combined features collected from imaging data (MRI biomarkers) with age and neuropsychological test results with a Random Forest (RF) classifier [31]. Ag- 
gregated biomarkers raised the classification accuracy by $5.5 \%$ (from $76.5 \%$ to $82 \%$ ). Grassi et al. [32] investigated sociodemographic information, clinical characteristics, neuropsychological measures of 550 subjects and combined the results using supervised ensemble learning. Each parameter from the multiple data was tested with 13 machine learning techniques, including hyperparameters optimization and cross-validation procedures. All the initially selected categorical features (14 continuous, 2 dichotomous, and 1 polytomous) were weighted, ranked, and organized into site-independent, stratified sub-sets. The most discriminative features were selected and contributed to the final result, which showed an AUROC (Area Under the Receiver Operating Characteristics) of 0.88 , a sensitivity of $77.7 \%$, and a specificity of $79.9 \%$.

Deep learning methods have received wide popularity in many domains including image processing and analysis. Their popularity is based on the fact that, in most cases, they did not require image preprocessing and feature engineering prior to the classification process. These algorithms work well with large and biased data. A convolutional neural network is a class of deep neural networks that was specially designed to work with imaging data. It can distinguish between AD and NC with an accuracy of $98 \%$ and predict a stable or converted $\mathrm{MCI}$ with an accuracy of $75 \%$ while processing and training only MRI data [33]. Other neural network models were proposed for the processing of non-imaging medical data. For example, Stamate et al. [34] created three deep learning models for processing and analysis of clinical and genetic data, and several parameters collected from MRI and PET images. Their models achieved $86 \%$ accuracy in the classification of dementia and cognitive impairment.

Table 1 summarizes state-of-the-art methods and their results for the diagnosis of Mild Cognitive Impairment and Alzheimer's Disease using the ADNI database.

Table 1. State-of-the-art methods of diagnosis of Mild Cognitive Impairment and Alzheimer's Disease.

\begin{tabular}{|c|c|c|}
\hline Authors & Methods & Results \\
\hline Lama et al. [26] & $\begin{array}{l}\text { PCA Features + Regularized Extreme } \\
\text { Learning Machine (unsupervised } \\
\text { classification learning algorithm based on } \\
\text { single hidden-layer feedforward neural } \\
\text { networks) of MRI (AD, MCI, NC). }\end{array}$ & $\begin{array}{l}\text { Accuracy: } 80.32 \% \text { (for binary } \\
\text { classification), } 76.61 \% \text { (for multiclass.) }\end{array}$ \\
\hline Zhou et al. [20] & $\begin{array}{l}\text { Transfer Learning Method (includes } \\
\text { Transfer AdaBoost algorithm) + } \\
\text { C4.5 classifier of MRI (AD, MCI, NC.) }\end{array}$ & $\begin{array}{l}\text { Accuracy: } 85.4 \% \text { (improves with } \\
\text { optimized feature selection). }\end{array}$ \\
\hline Beheshti et al. [21] & $\begin{array}{l}\text { Feature-ranking + genetic algorithm + } \\
\text { SVM classifier of MRI (AD, MCI). }\end{array}$ & $\begin{array}{c}\text { Accuracy: } 93.01 \% \text { (stable MCI), } 75 \% \\
\text { (progressive } \mathrm{MCI} \text { ), } 78.94 \% \text { (without } \\
\text { feature selection), } 94.73 \% \text { (with } \\
\text { feature selection). }\end{array}$ \\
\hline Moradi et al. [30] & $\begin{array}{l}\text { Logic regression + MRI biomarker (based } \\
\text { on low-density separation) + SVM + } \\
\text { neuropsychological test results + random } \\
\text { forest classifier of MRI (AD, MCI, NC). }\end{array}$ & $\begin{array}{l}\text { MRI + cognitive test improves the } \\
\text { accuracy by } 5.5 \% \text { (from } 76.5 \% \text { to } 82 \% \text { ). }\end{array}$ \\
\hline Glozman and Le [28] & $\begin{array}{l}\text { Feature ranking of the white matter (WM) } \\
+ \text { SVM (with Linear and RBF Kernels) } \\
\text { and Logic Regression of DTI (AD). }\end{array}$ & Average accuracy: 92\%. \\
\hline Grassi et al. [32] & $\begin{array}{l}\text { Ensemble algorithm using } \\
\text { sociodemographic information, } \\
\text { clinical characteristics, neuropsychologi- } \\
\text { cal measures; supervised ML. } \\
\text { (Conversion from MCI to AD). }\end{array}$ & $\begin{array}{l}\text { AUROC: } 0.88 \text {; sensitivity: } 77.7 \% \text {; } \\
\text { specificity: } 79.9 \% . \\
\text { Range of AUROC for proposed } \\
\text { models is } 0.83-0.90 .\end{array}$ \\
\hline Basaia et al. [33] & $\begin{array}{l}\text { CNNs; classification of } \mathrm{AD} \text {, stable MCI } \\
\text { and converted MCI. Did not use } \\
\text { feature engineering. }\end{array}$ & $\begin{array}{l}\text { Accuracy of AD vs. CN: } 98 \% \text {; } \\
\text { sMCI vs. cMCI: } 75 \% \text {. }\end{array}$ \\
\hline
\end{tabular}


Table 1. Cont.

\begin{tabular}{ccc}
\hline Authors & Methods & Results \\
\hline & Deep Learning models: two Multi-Layer & \\
& Perceptron (MLP1 and MLP2) models & \\
and a Convolutional & The best models (MLP1 and MLP2) \\
Stamate et al. [34] & Bidirectional Long Short- & show the accuracy 0.86 for Dem, MCI, \\
& Term Memory (ConvBLSTM) model. & and classes. \\
& The features were collected from clinical & \\
and genetic data, MRI data, PET data and & some additional biospecimen. \\
(Dem, MCI, CN).
\end{tabular}

In contrast with previous work (see Table 1), this study proposes a new approach to the early diagnosis of dementia. The pipeline includes an asymmetry segmentation algorithm that visualizes the differences between the right and the left hemispheres of the MRI slices of the brain. The features are collected directly from the regions that have been already affected by the degenerative atrophic processes. This approach simplifies the feature engineering stage and gives an advantage over other state-of-the-art methods mentioned above, where feature collection and selection processes are more complicated and time-consuming. The images of asymmetries take less memory space than original MRIs. For example, two images (original and asymmetry) in the same file type and dimensions have sizes of 55.6 KB and 18.7 KB, respectively. This reduces the storage of the imaging data and speeds up the classification processing of large datasets that make use of images as an input. The proposed method, unlike the methods above, does not require special hardware equipment and cloud computations. Experiments, presented in detail in Section 4, provide evidence that it performs well even without fine-tuning the classification models. For example, the prediction of early mild cognitive impairment compared to the normal controls using SVM is $92.5 \%$, which appears quite promising compared with the accuracy of other fine-tuned models that is in the range between $80.32 \%$ and $92 \%$, as shown in Table 1.

\section{Materials and Methods}

\subsection{Data Repositories and Participants}

Data used in the preparation of this article were obtained from the Alzheimer's Disease Neuroimaging Initiative (ADNI) database (adni.loni.usc.edu), which was launched in 2003 as a public-private partnership led by Michael W. Weiner, MD. The primary goal of ADNI has been to test whether serial magnetic resonance imaging (MRI), positron emission tomography (PET), other biological markers, and clinical and neuropsychological assessment can be combined to measure the progression of mild cognitive impairment (MCI) and early Alzheimer's disease (AD); for up-to-date information, see www.adni-info.org (Supplementary Materials).

The datasets of T1-waited images have been created using MRI data of 750 subjects aged between 55 and 75 years. Patients are divided equally into groups of subjects with normal cognition (NC), early mild cognitive impairment (EMCI), and Alzheimer's Disease (AD).

\subsection{Research Methods}

The data processing pipeline, including image processing and machine learning classification, has been implemented in Matlab using affordable and easy-to-obtain commodity hardware. The supported software and hardware characteristics are as follows: Windows 10 Enterprise, processor-Intel (R) Core (TM), i7-7700 CPU@ 3.60 GHz, 16 GB RAM.

The methods for early diagnosis of dementia include detection of image asymmetries, statistical feature extraction, and image analysis, machine learning algorithms. 
Figure 1 illustrates the stages of data processing, analysis, and classification of the pipeline, whilst the dashed line accentuates the contribution of this paper.

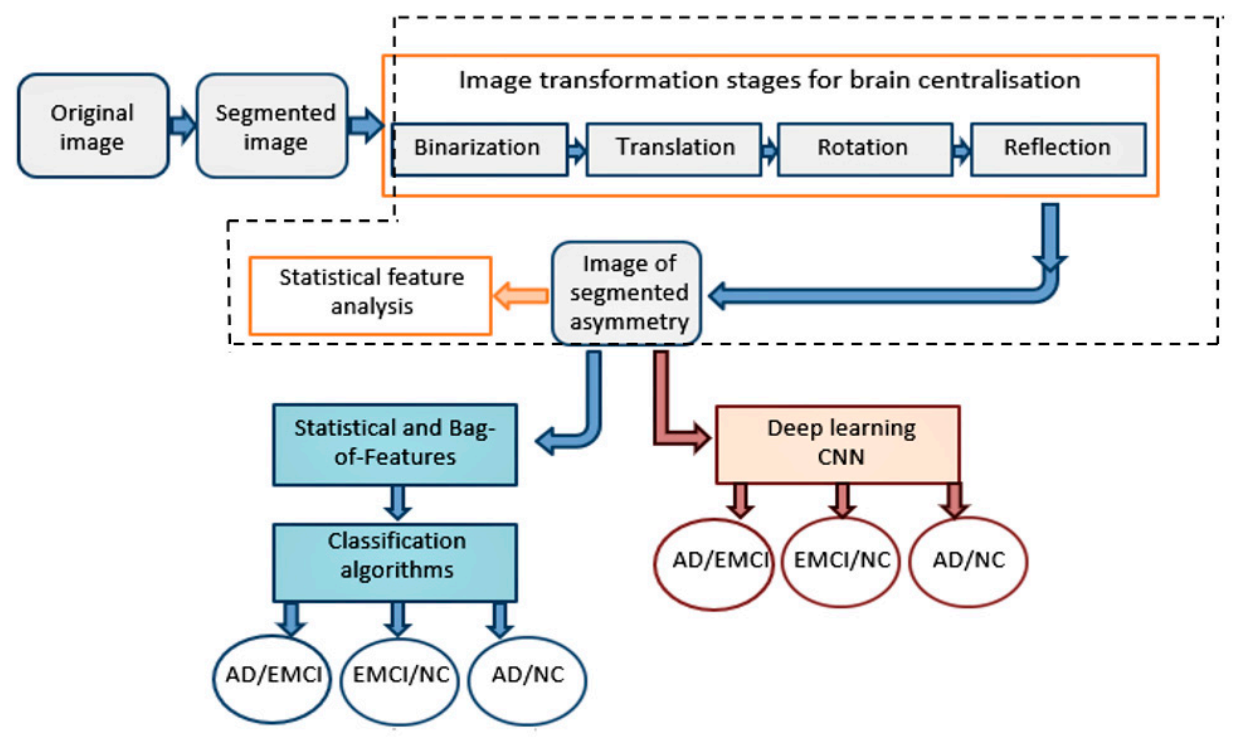

Figure 1. The MRI data processing pipeline includes image transformation stages with the following feature extraction and machine learning classifications.

\subsubsection{Image Preprocessing}

All images in the dataset were normalized and resized to 256-by-256-by-3 pixels. Then, brain tissues were segmented manually from the skull using adjusted upper and lower boundaries of a threshold level of the pixel values. This simple algorithm can be replaced with higher0quality brain segmentation software [35,36].

\subsubsection{Detection of Image Asymmetry}

A key stage of the pipeline is the detection of the vertical line of symmetry in the brain. As soon as that line is found, it is possible to flip the brain image from the left to the right across the vertical axis and extract the image asymmetries. A flipped or reverse image is an image that is mirrored across the horizontal or vertical axis [37].

The hypothesis being tested in this part of the research is that there is an axis of reflective symmetry running through the center of the brain [38]. Thus, it is necessary to find the center of the brain and translate it to the center of the image. At the same time, the brain needs to be rotated to the correct angle to reach the maximum symmetry in the image. The algorithm was tested on single slices of the brain. The same idea can be extended and applied to the whole 3D brain.

The brain center is allocated using an image binarization technique and calculating the image centroid [39]. Image binarization [40] is the process of converting a grayscale image to black and white. As a result, 256 shades of the grayscale image are reduced to 2 colors only. The binarization is done according to the level of a threshold. All pixels in the image above the threshold level are replaced by the value 1 (white) and other pixels that are below that level by the value 0 (black).

In the context of image processing and computer vision, the centroid is the weighted average of all the pixels in an image. The "weighted" centroid, or center of mass, is always at the exact center and depends on the gray levels in the image.

Figure 2 illustrates the main stages of the image processing prior to classification. 


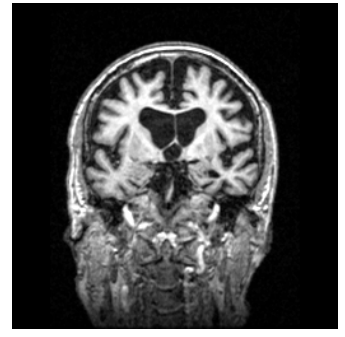

(a)

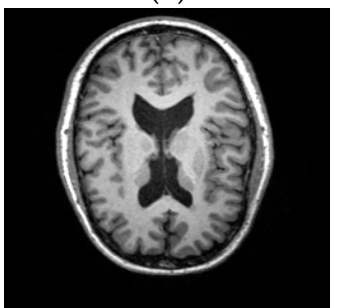

(d)

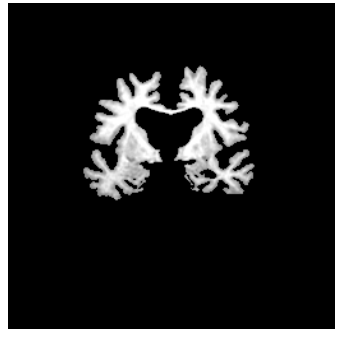

(b)

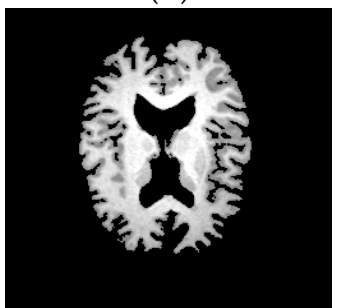

(e)

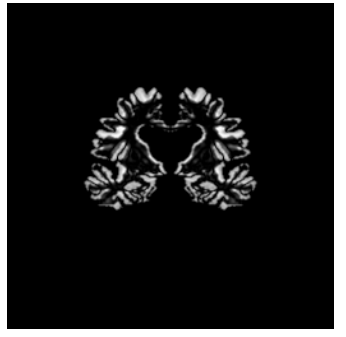

(c)

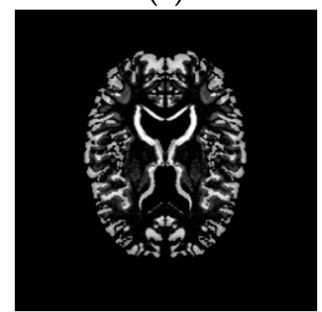

(f)

Figure 2. T1-waited brain MRI processing in 2 axes of symmetries: (a) Original image in the coronal plane; (b) Segmented image of the brain tissues in the coronal plane; (c) Image asymmetry in the coronal plane; (d) Original image in the axial plane; (e) Segmented image of the brain tissues in the axial plane; (f) Image asymmetry in the axial plane.

Detailed image transformation stages are provided in Figure 3.

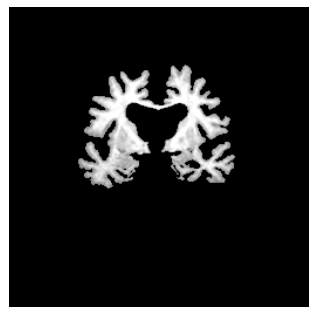

(a)

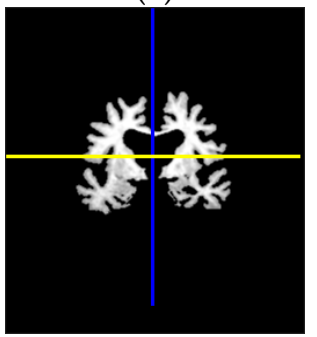

(d)

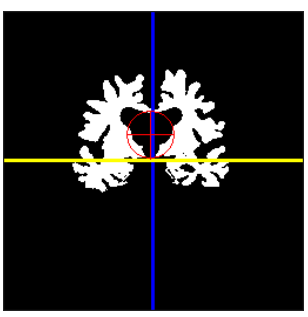

(b)

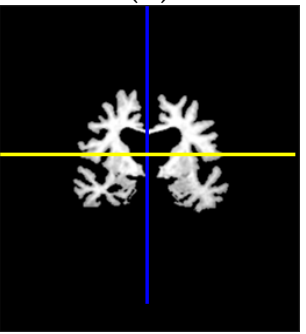

(e)

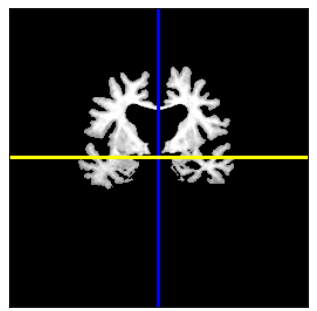

(c)

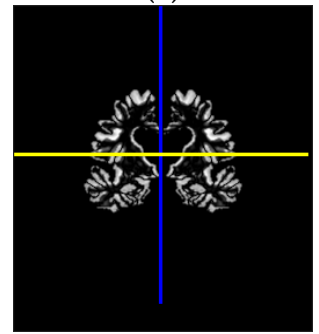

$(\mathbf{f})$

Figure 3. Image transformation stages: (a) Segmented grayscale image; (b) Centers of the binary image; (c) Image translated to the middle of the vertical axis; (d) Centered and rotated image with respect to 2 axes of symmetry; (e) Reflected image via vertical axis; (f) Image asymmetry.

The last image is obtained as a result of the mirroring of the left-brain hemisphere to the right and right-brain hemisphere to the left, which is followed by subtraction of the hemispheres from each other. This process can be expressed symbolically as an equation:

$$
\mathrm{D}=(\mathrm{L}-\mathrm{R})+(\mathrm{R}-\mathrm{L})
$$

where $\mathrm{D}$ is an image asymmetry, $\mathrm{L}$ is an image matrix of the left hemisphere, $\mathrm{R}$ is an image matrix of the right hemisphere. 
Figure 4 illustrates the pixel transformation values from segmentation of asymmetry in a small image of size 6-by-6. The numbers in the cells correspond to the gray level of the pixel values.

\begin{tabular}{|l|l|l|l|l|l|}
\hline 0 & 0 & 20 & 10 & 0 & 0 \\
\hline 0 & 0 & 35 & 100 & 140 & 0 \\
\hline 0 & 87 & 17 & 17 & 85 & 0 \\
\hline 0 & 56 & 120 & 120 & 17 & 0 \\
\hline 61 & 30 & 96 & 96 & 30 & 0 \\
\hline 210 & 42 & 15 & 15 & 10 & 0 \\
\hline
\end{tabular}

(a)

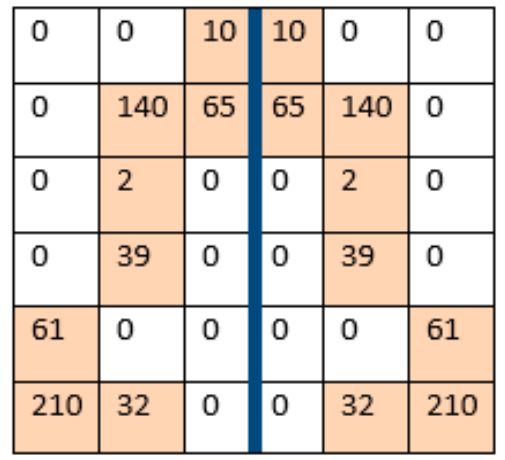

(b)

Figure 4. Matrices of gray-scale image: (a) Initial matrix; (b) Matrix of segmented asymmetry mirrored via the vertical axis.

The symmetrical image areas (Figure $4 \mathrm{~b}$ ) become equal to 0 due to matrix subtraction. They are visualized as black areas in the image. The asymmetrical parts of the image are represented as different intensity gray levels from 1 to 255 .

\subsubsection{Generating Asymmetry Features}

The statistical feature approach for representing image properties is well-known in image processing [41]. Extracted statistical features represent the color, texture, or morphological properties of an image. Ten strong and stable statistical features, namely MSE (Mean Squared Error), Mean, Std (Standard deviation), Entropy, RMS (Root Mean Square), Variance, Smoothness, Kurtosis, Skewness, and IDM (Inverse difference moment) [42-48], are chosen for the research and combined in vectors representing the MRI data properties. These features give information about the likelihood of gray pixel values in a random position in an image, their orientation, and interaction with other surrounding pixels [24]. In our pipeline, images with segmented asymmetry have been analyzed to generate statistical features from the image differences using discrete wavelet transform (DWT) [49,50]. The only exception is the first feature on the list, Mean Squared Error, which has been calculated as a difference between the original image and its mirrored version without wavelet transformation.

As an example, the features vector, extracted from the image asymmetry of a patient's MRI slice, is provided below:

[849.477703 10.47024065 90.10031233 0.76467623951.73779963 7260.005288 0.999995013 52.782242775.661092548 9052.865381]

Figure 5 shows features averaging based on normalized (from 0 to 1 ) features data extracted from a set of 300 images with segmented asymmetry, which were generated from a set of $300 \mathrm{MRI}$ slices from different patients equally distributed in each group.

Figure 6 provides an MSE feature analysis with a Pareto chart. The MSE value for each class has been calculated from the differences between the original image and its mirrored version for all images in that class and indicates the impact of asymmetry for each class. The highest MSE bar for AD confirms that changes in symmetry in the MRI slices of this image group are substantial compared to changes in symmetry in the MRI slices of the other groups, with the EMCI group's images looking more "symmetrical" than the others. The cumulative line on the secondary axis shows the contribution of each bar (image class) in the total value as a percentage. 


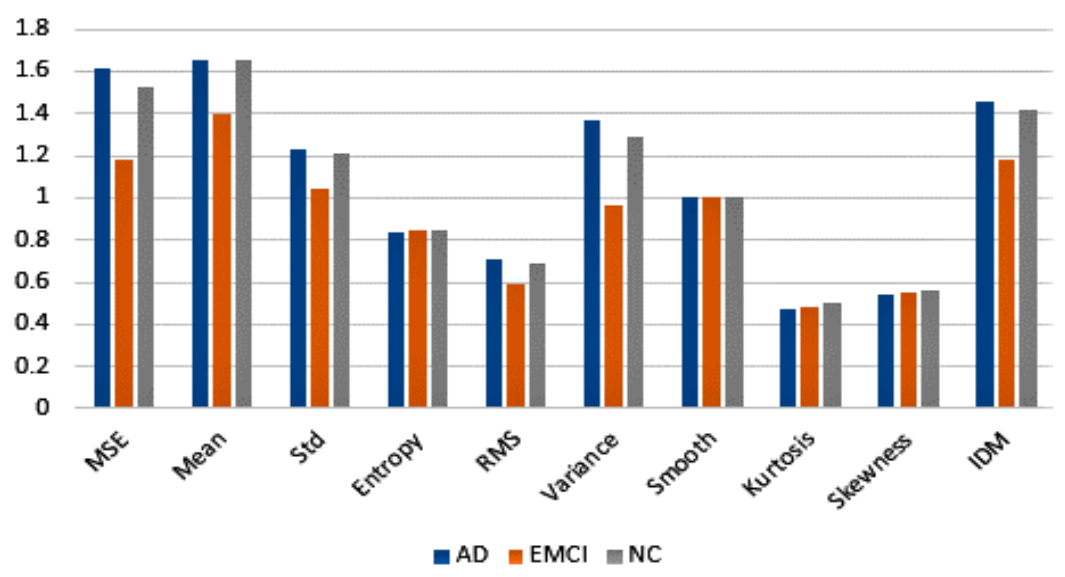

Figure 5. Statistical mean of each image asymmetry feature for normal cognition (NC), early minor cognitive impairment (EMCI), and Alzheimer's disease (AD) patients.

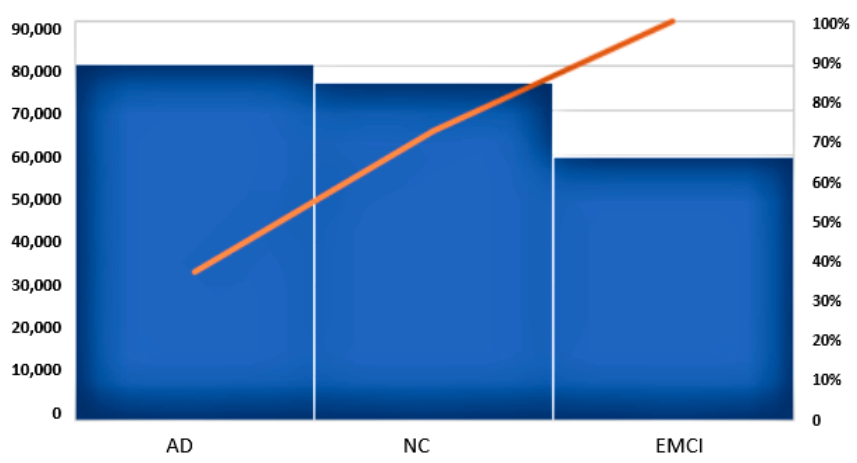

Figure 6. Pareto chart of MSE for the three classes. The primary axis, on the left, shows the total MSE feature value, whilst the secondary axis, on the right, shows the cumulative percentage of the total value for each class.

Further analysis of image asymmetries based on 10 statistical features (Figure 5) confirms that the above observations are present in other datasets and can be extended to different sizes of data (MRI slices). These findings support the view that image asymmetry decreases in the initial stage of the generative process in the brain (Early Mild Cognitive Impairment) and grows when the person develops moderate and severe dementia (Alzheimer's disease).

Statistical features collected from image asymmetries were enriched with Bag-ofFeatures (BOF) to get the most detailed image "signatures". State-of-the-art literature confirms the high performance of the Bag-of-Features algorithm in image classification [51]. BOF [52] based on the Speeded Up Robust Features (SURF) [53,54] and the K-Means clustering [55] algorithms were represented as vectors of occurrence of the local image features in this work.

\subsubsection{Classification Using Machine Learning}

In this stage of the pipeline, various machine learning methods were trialed as reported in the next section. The purpose was to verify the robustness of brain asymmetry image and asymmetry features for early diagnosis of dementia when applied to different classification models without fine-tuning their hyperparameters. Supervised machine learning was the main focus, and the following binary classifiers were used: Naïve Bayes (NB) [56], Linear Discriminant (LD) [57], Support Vector Machine (SVM) (linear, quadratic, cubic, Medium Gaussian kernels) [58,59], K-Nearest Neighbor (fine, cosine kernels) [60]. All models from the list were trained using Matlab Classification Learner App, except the 
NB classifier, which was trained separately. Table 2 presents the models default hyperparameter values used in the experiments.

Table 2. Hyperparameters of the machine learning algorithms.

\begin{tabular}{|c|c|}
\hline Model & Hyperparameters \\
\hline NB & Distribution: normal (Gaussian) \\
\hline LD & Discriminant type: linear \\
\hline L-SVM & $\begin{array}{l}\text { Kernel function: linear } \\
\text { Box constraint level:1 } \\
\text { Kernel scale mode: auto } \\
\text { Standardize data: true }\end{array}$ \\
\hline Q-SVM & $\begin{array}{l}\text { Kernel function: quadratic } \\
\text { Box constraint level:1 } \\
\text { Kernel scale mode: auto } \\
\text { Standardize data: true }\end{array}$ \\
\hline C-SVM & $\begin{array}{l}\text { Kernel function: cubic } \\
\text { Box constraint level:1 } \\
\text { Kernel scale mode: auto } \\
\text { Standardize data: true }\end{array}$ \\
\hline MG-SVM & $\begin{array}{c}\text { Kernel function: medium Gaussian } \\
\text { Box constraint level:1 } \\
\text { Kernel scale mode: manual } \\
\text { Kernel scale: } 32 \\
\text { Standardize data: true }\end{array}$ \\
\hline Fine-KNN & $\begin{array}{c}\text { Number of neighbors: } 1 \\
\text { Distance metric: Euclidian } \\
\text { Distance weight: equal } \\
\text { Standardize data: true }\end{array}$ \\
\hline Cos-KNN & $\begin{array}{l}\text { Number of neighbors: } 10 \\
\text { Distance metric: cosine } \\
\text { Distance weight: equal } \\
\text { Standardize data: true }\end{array}$ \\
\hline
\end{tabular}

The potential of transfer learning was also explored by applying a type of Convolutional Neural Network (CNN), the so-called AlexNet [61,62]. This is a convolutional neural network with 8 deep layers. The network has five convolutional layers and three fully connected layers. The 1-st layer requires input image of size 227-by-227-by-3, where 3 is the number of color channels.

Figure 7 displays the network architecture. The last 3 layers of AlexNet were preliminary configured to 1000 classes as it was trained to solve a different classification problem. To adopt the network for the current classification task, the last 3 layers of the AlexNet were replaced with a fully connected layer, a softmax layer, and a binary classification output layer.

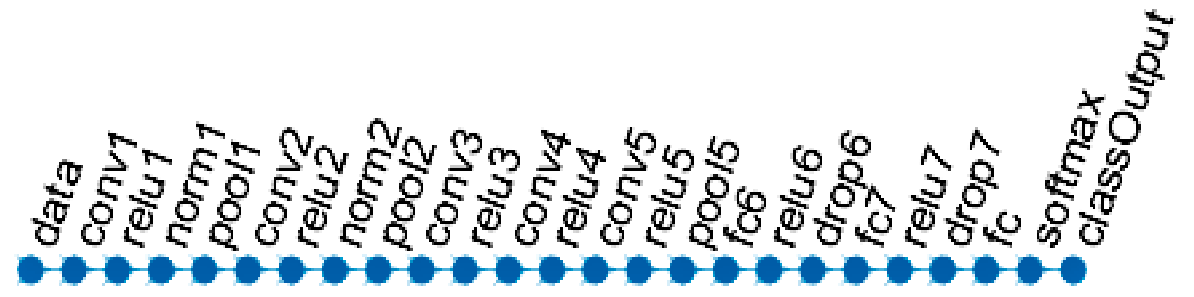

Figure 7. Adapted AlexNet architecture used in the experiments. 


\section{Experiments and Results}

Six hundred images of brain asymmetries with equal number of $\mathrm{AD}, \mathrm{EMCI}$, and $\mathrm{NC}$ subjects were combined into three binary datasets, EMCI vs. NC, AD vs. NC, and AD vs. EMCI. The datasets include images in 2 dimensions (planes): vertical or frontal and horizontal or axial. The purpose of the experiment was to investigate whether asymmetry features produce consistent performance across different classification models, and no fine-tuning or model optimization was performed.

The performance of NB, LD, SVM, and KNN trained models was estimated using 10 simulation runs of a 10-fold cross-validation procedure, while for the adapted AlexNet $(\mathrm{CNN}), 2$ simulation runs were conducted without using cloud infrastructure. Training and evaluation of the $\mathrm{CNN}$ are also processed differently from the rest of methods: images of segmented asymmetry were resized to $227 \times 227 \times 3$ and fed into the model with $80 \%$ of the images used for training, $10 \%$ for validation, and $10 \%$ for testing. The parameters of the CNN were 128 mini-batch size, 10 epochs, validation data frequency of 50 . Each CNN training/validation/testing round took approximately 43 min on our hardware, while SVMs were trained for 10 epochs, with each epoch taking approximately $5 \mathrm{~s}$. All the machine learning models were tested on unseen data, and Table 3 summarises the average performance (\%) in testing of the early mild cognitive impairment, normal cognition, and Alzheimer's disease datasets of the ADNI database. The highest results for each dataset are shown in bold.

Table 3. Average performance (\%) of binary classifiers.

\begin{tabular}{cccccccccc}
\hline Datasets & NB & LD & L-SVM & Q-SVM & C-SVM & MG-SVM & Fine-KNN & Cos-KNN & CNN \\
\hline EMCI vs. NC & & & & & & & & & \\
Accuracy & 77.0 & 91.0 & 89.0 & $\mathbf{9 2 . 5}$ & $\mathbf{9 2 . 5}$ & 88.0 & 83.0 & 92.0 & 75.0 \\
Sensitivity & 78.0 & 91.0 & 89.0 & 92.0 & 95.0 & 85.0 & 99.0 & 96.0 & 90.0 \\
Specificity & 76.0 & 91.0 & 89.0 & 93.0 & 90.0 & 91.0 & 67.0 & 88.0 & 60.0 \\
\hline AD vs. NC & & & & & & & & & \\
Accuracy & 78.5 & 90.0 & 92.0 & 92.5 & $\mathbf{9 3 . 0}$ & 90.0 & 86.5 & 89.5 & 90.0 \\
Sensitivity & 78.0 & 88.0 & 91.0 & 90.0 & 93.0 & 85.0 & 98.0 & 90.0 & 89.0 \\
Specificity & 79.0 & 92.0 & 93.0 & 95.0 & 93.0 & 95.0 & 75.0 & 89.0 & 92.0 \\
\hline AD vs. EMCI & & & & & & & & & \\
Accuracy & 78.5 & 83.0 & 80.5 & 86.5 & $\mathbf{8 6 . 5}$ & 80.5 & 79.0 & 80.0 & 81.25 \\
Sensitivity & 75.0 & 85.0 & 84.0 & 89.0 & 88.0 & 84.0 & 78.0 & 83.0 & 72.5 \\
Specificity & 81.0 & 81.0 & 77.0 & 84.0 & 85.0 & 77.0 & 80.0 & 78.0 & 90.0 \\
\hline
\end{tabular}

The test results show that features extracted from asymmetries provide consistent performance across different classification models without model-specific fine-tuning of hyperparameters. The SVM variants and the LD method can become the methods of choice as they can be easily trained on commodity hardware and demonstrate better accuracy than other alternatives in all cases. The best performance amongst the SVM variants was shown by SVMs with the polynomial cubic and quadratic kernel (C-SVM and Q-SVM). C-SVM accuracy of EMCI vs. NC was $92.5 \%$, sensitivity was $95.0 \%$, specificity was $90.0 \%$; accuracy of AD vs. NC was $93.0 \%$, sensitivity was $93.0 \%$, specificity was $93.0 \%$; accuracy of AD vs. EMCI was $86.5 \%$, sensitivity was $88.0 \%$, specificity was $85.0 \%$. Q-SVM accuracy of EMCI vs. NC was $92.5 \%$, sensitivity was $92.0 \%$, specificity was $93.0 \%$; accuracy of AD vs. NC was $92.5 \%$, sensitivity was $90.0 \%$, specificity was $95.0 \%$; accuracy of AD vs. EMCI was $86.5 \%$, sensitivity was $89.0 \%$, specificity was $84.0 \%$. The prediction results of the $\mathrm{CNN}$ are in the same range as those of the other classifiers.

An aggregated measure, the area under the ROC Curve (AUC), shows the relationship between data sensitivity and specificity across different levels of threshold [63], giving an additional view on the classifier performance. AUC results of the best available models from C-SVM and CNN are presented in Table 4. 
Table 4. AUC for cubic-SVM and CNN.

\begin{tabular}{ccc}
\hline Datasets & C-SVM & CNN \\
\hline EMCI vs. NC & 0.98 & 0.90 \\
AD vs. NC & 0.99 & 0.92 \\
AD vs. EMCI & 0.94 & 0.88 \\
\hline
\end{tabular}

Figure 8 presents the AUC/ROC curves of three binary datasets for the C-SVM and $\mathrm{CNN}$ classifiers. All the sub-figures were created on Matlab during data testing.

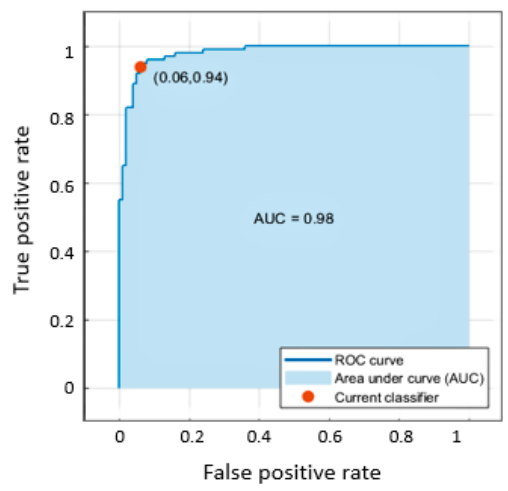

(a)

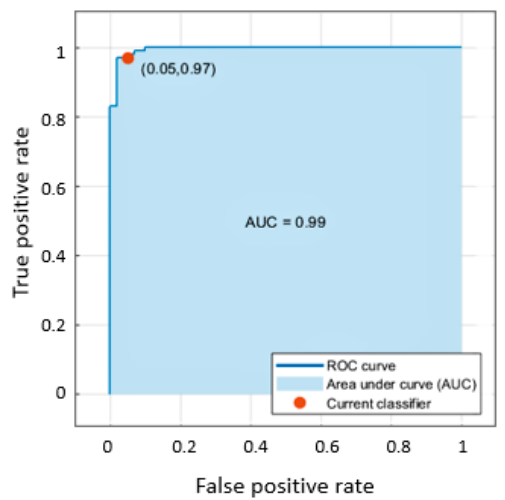

(c)

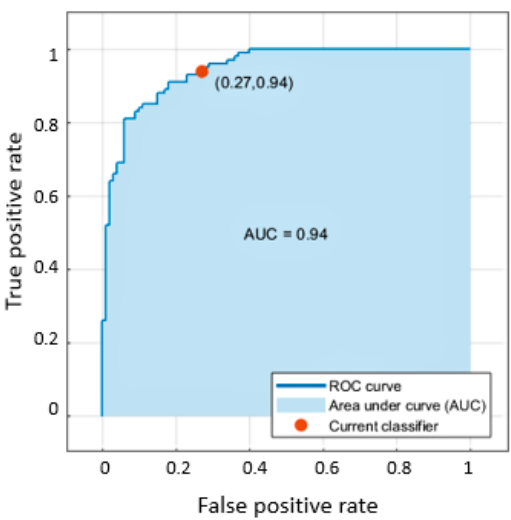

(e)

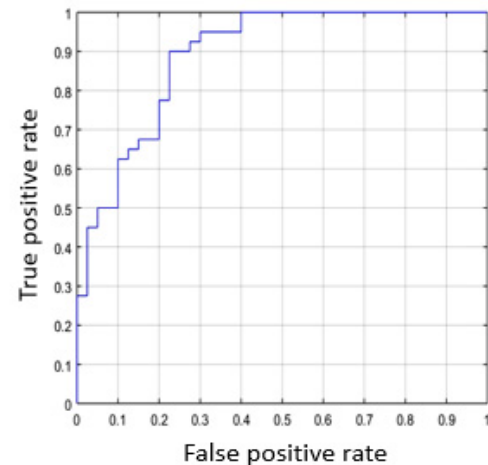

(b)

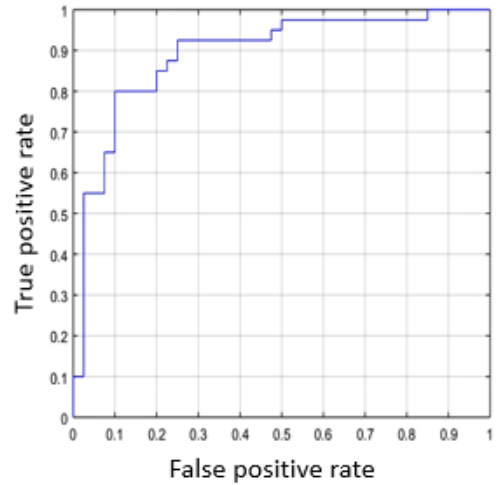

(d)

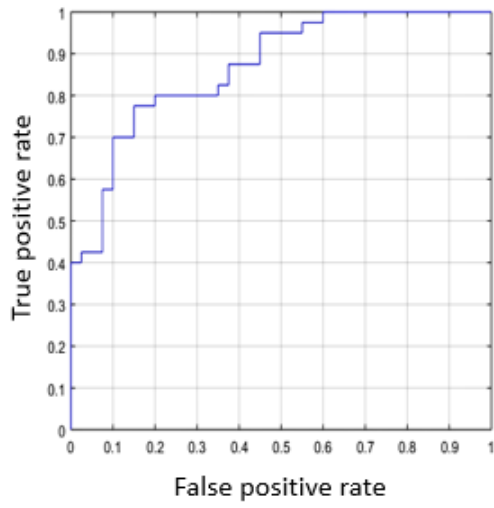

(f)

Figure 8. AUC/ROC curves of Cubic Support Vector Machine (C-SVM) (left) and Convolutional Neural Network (CNN) (right) classifiers: $(\mathbf{a}, \mathbf{b})$ is EMCI vs. NC; $(\mathbf{c}, \mathbf{d})$ is AD vs. NC; $(\mathbf{e}, \mathbf{f})$ is AD vs. EMCI. 
In general, satisfactory performance was obtained in classification between EMCI and NC, AD and NC, and AD and EMCI. In this context, it is worth noting an important difference between the CNN classifier and all other methods: the CNN is the only model that operates directly on images of segmented asymmetry, whilst all other models operate on images with segmented asymmetry that have been analyzed to generate statistical features using discrete wavelet transform (DWT). It is expected that fine-tuning and model optimization can potentially improve the performance of all classification models further. This will be the subject of another communication of ours, since the focus of the current study has been on demonstrating the potential of brain asymmetry images and features. In the next section, the classification performance using asymmetry features is discussed further in the context of the literature on the diagnosis of dementia.

\section{Discussion and Conclusions}

The paper introduced a new diagnostic approach based on analysis of brain asymmetry for early classification of initial dementia when clinical symptoms are very mild and challenging. The early changes in the brain asymmetries in subjects with early mild cognitive impairment are accompanied by an increase in the symmetry between the left and the right hemisphere. The proposed pipeline is based on the detection of the image asymmetries using magnetic resonance imaging. The study involved the imaging resources of the well-known ADNI medical imaging database. The experimental study presented in the previous section included cross-sectional classification of early mild cognitive impairment, Alzheimer's disease, and cognitively normal subjects from the ADNI database. The experimental results support the hypothesis that changes in the brain asymmetries during the development of dementia convey important information (see [5]), as they were used to generate useful features for classification.

In contrast to other methods in the literature (see Section 2), this study is less complex in term of image processing. For example, the image processing time on average was 0.1 min per image (3.6 GHz Intel Core i7, 16 GB RAM), compared to the article [30], where it took $8 \mathrm{~min}$ (3.4 GHz Intel Core i7, $8 \mathrm{~GB}$ RAM). The images of segmented asymmetries require 3 times less memory space than similar originals. This potentially gives an advantage in terms of computational time spent on training a classifier, although it is not possible to make a direct comparison with other approaches in the literature as hardware specifications differ. Nevertheless, it is worth mentioning that the CPU time for an asymmetry features-trained single classifier, including 10 -fold cross-validation, was approximately $5 \mathrm{~s}$ on our hardware, which appears considerably low compared to other methods that used more complex feature sets [31]. Classification performance achieved in this paper is comparable with the results obtained by other researchers, summarized in Table 1 for the ADNI database. In particular, predictive accuracy of $92.5 \%$ for EMCI vs. $\mathrm{CN}$ and $93 \%$ for $\mathrm{AD}$ vs. $\mathrm{CN}$ is high, especially when considering the complexity of the schemes shown in this table (e.g., a larger number of features, a higher number of architectural parameters, and so on). Further performance improvement can be potentially achieved with SVM and CNN classifiers by optimization of architectures, as demonstrated in other works in the literature [29,32]; a study of this type is included in our immediate plans.

A limitation of this study is that it did not investigate handedness and its influence on the classification results, as the images downloaded from the ADNI database were not separated according to the handedness. Thus, a potential direction for further work concerns an investigation of the role and the impact of asymmetric differences between the right- and left-handed patients. In addition, the potential of deep learning models deserves further study. Lastly, a longitudinal study inside the group of patients with mild cognitive impairment could help to distinguish between stable and progressive dementia and monitor further changes in the brain due to the development of the disease. This asymmetrical changes in the brain can be tested with other psychiatric conditions such as schizophrenia and Parkinson's Disease. 
On a practical level, the use of image asymmetry and asymmetry features has potential to contribute to the design of end-user (e.g., physicians and general medical practitioners) applications, which will run on commodity hardware, for early diagnosis of cognitive decline and investigation of the nature of the structural changes in the brain. These applications can exploit the generalization properties of machine learning models on unseen MRI data, as demonstrated in this study.

Supplementary Materials: The MRI images are available online at www.adni-info.org. The source code is available at https://uk.mathworks.com/matlabcentral/fileexchange/85628-asymmetrydetection-of-the-mri-brain.

Author Contributions: Conceptualization, N.J.H.; software, N.J.H.; validation, N.J.H.; writingoriginal draft preparation, N.J.H.; writing — review and editing, G.D.M.; supervision, G.D.M. All authors have read and agreed to the published version of the manuscript.

Funding: The authors gratefully acknowledge financial support by Birkbeck College, University of London, through a BEI School Award.

Institutional Review Board Statement: This study did not involve patients. The MRI data collection procedures were approved by the institutional review boards of all participating centers to the Alzheimer's Disease Neuroimaging Initiative.

Informed Consent Statement: Not applicable for this study. Participating centers to the Alzheimer's Disease Neuroimaging Initiative obtained written informed consent from all participants or their authorized representatives for MRI data collection.

Data Availability Statement: The MRI images used in the preparation of this article were obtained from the ADNI database (adni.loni.usc.edu), which is available for download from the Laboratory of Neuroimaging (LONI). Data use is subject to an Alzheimer's Disease Neuroimaging Initiative (ADNI) data use agreement.

Acknowledgments: Data collection and sharing for this project was funded by the Alzheimer's Disease Neuroimaging Initiative (ADNI) (National Institutes of Health Grant U01 AG024904) and DOD ADNI (Department of Defense award number W81XWH-12-2-0012). ADNI is funded by the National Institute on Aging, the National Institute of Biomedical Imaging and Bioengineering, and through generous contributions from the following: AbbVie, Alzheimer's Association; Alzheimer's Drug Discovery Foundation; Araclon Biotech; BioClinica, Inc.; Biogen; Bristol-Myers Squibb Company; CereSpir, Inc.; Cogstate; Eisai Inc.; Elan Pharmaceuticals, Inc.; Eli Lilly and Company; EuroImmun; F. Hoffmann-La Roche Ltd. and its affiliated company Genentech, Inc.; Fujirebio; GE Healthcare; IXICO Ltd.; Janssen Alzheimer Immunotherapy Research \& Development, LLC.; Johnson \& Johnson Pharmaceutical Research \& Development LLC.; Lumosity; Lundbeck; Merck \& Co., Inc.; Meso Scale Diagnostics, LLC.; NeuroRx Research; Neurotrack Technologies; Novartis Pharmaceuticals Corporation; Pfizer Inc.; Piramal Imaging; Servier; Takeda Pharmaceutical Company; and Transition Therapeutics. The Canadian Institutes of Health Research is providing funds to support ADNI clinical sites in Canada. Private sector contributions are facilitated by the Foundation for the National Institutes of Health (www.fnih.org). The grantee organization is the Northern California Institute for Research and Education, and the study is coordinated by the Alzheimer's Therapeutic Research Institute at the University of Southern California. ADNI data are disseminated by the Laboratory for Neuro Imaging at the University of Southern California.

Conflicts of Interest: The authors declare no conflict of interest.

\section{References}

1. Thabtah, F.; Mampusti, E.; Peebles, D.; Herradura, R. A mobile-based screening system for data analyses of early dementia traits detection. J. Med. Syst. 2020, 44, 24. [CrossRef] [PubMed]

2. Yanase, J.; Triantaphyllou, E. A systematic survey of computer-aided diagnosis in medicine: Past and present developments. Expert Syst. Appl. 2019, 138, 112821. [CrossRef]

3. Lazli, L.; Boukadoum, M.; Mohamed, O.A. A Survey on Computer-Aided Diagnosis of Brain Disorders through MRI Based on Machine Learning and Data Mining Methodologies with an Emphasis on Alzheimer Disease Diagnosis and the Contribution of the Multimodal Fusion. Appl. Sci. 2020, 10, 1894. [CrossRef]

4. Toga, A.W.; Thompson, P.M. Mapping brain asymmetry. Nat. Rev. Neurosci. 2003, 4, 37. [CrossRef] [PubMed] 
5. McManus, C. Half a century of handedness research: Myths, truths; fictions, facts; backwards, but mostly forwards. Brain Neurosci. Adv. 2019, 3. [CrossRef] [PubMed]

6. Bitar, M.; Barry, G. Multiple innovations in genetic and epigenetic mechanisms cooperate to underpin human brain evolution. Mol. Biol. Evol. 2018, 35, 263-268. [CrossRef]

7. Isles, A.R. Epigenetics, chromatin and brain development and function. Brain Neurosci. Adv. 2018, 2. [CrossRef]

8. Tomasi, D.; Volkow, N.D. Laterality patterns of brain functional connectivity: Gender effects. Cereb. Cortex 2012, 22, 1455-1462. [CrossRef]

9. Cabeza, R. Hemispheric asymmetry reduction in older adults: The HAROLD model. Psychol. Aging 2002, 17, 85. [CrossRef]

10. Cabeza, R.; Daselaar, S.M.; Dolcos, F.; Prince, S.E.; Budde, M.; Nyberg, L. Task-independent and task-specific age effects on brain activity during working memory, visual attention and episodic retrieval. Cereb. Cortex 2004, 14, 364-375. [CrossRef]

11. Kalavathi, P.; Senthamilselvi, M.; Prasath, V.B. Review of computational methods on brain symmetric and asymmetric analysis from neuroimaging techniques. Technologies 2017, 5, 16. [CrossRef]

12. Yang, C.; Zhong, S.; Zhou, X.; Wei, L.; Wang, L.; Nie, S. The abnormality of topological asymmetry between hemispheric brain white matter networks in Alzheimer's disease and mild cognitive impairment. Front. Aging Neurosci. 2017, 9, 261. [CrossRef] [PubMed]

13. Liu, H.; Zhang, L.; Xi, Q.; Zhao, X.; Wang, F.; Wang, X.; Men, W.; Lin, Q. Changes in brain lateralization in patients with mild cognitive impairment and Alzheimer's disease: A resting-state functional magnetic resonance study from Alzheimer's disease neuroimaging initiative. Front. Neurol. 2018, 9, 3. [CrossRef] [PubMed]

14. Kim, J.H.; Lee, J.W.; Kim, G.H.; Roh, J.H.; Kim, M.J.; Seo, S.W.; Kim, S.T.; Jeon, S.; Lee, J.M.; Heilman, K.M.; et al. Cortical asymmetries in normal, mild cognitive impairment, and Alzheimer's disease. Neurobiol. Aging 2012, 33, 1959-1966. [CrossRef] [PubMed]

15. Wachinger, C.; Salat, D.H.; Weiner, M.; Reuter, M. Alzheimer's Disease Neuroimaging Initiative. Whole-brain analysis reveals increased neuroanatomical asymmetries in dementia for hippocampus and amygdala. Brain 2016, 139, 3253-3266. [CrossRef] [PubMed]

16. Wachinger, C.; Golland, P.; Kremen, W.; Fischl, B.; Reuter, M. Alzheimer's Disease Neuroimaging Initiative. BrainPrint: A discriminative characterization of brain morphology. NeuroImage 2015, 109, 232-248. [CrossRef] [PubMed]

17. Despotović, I.; Goossens, B.; Philips, W. MRI segmentation of the human brain: Challenges, methods, and applications. Comput. Math. Methods Med 2015. [CrossRef] [PubMed]

18. Zheng, A.; Casari, A. Feature Engineering for Machine Learning: Principles and Techniques for Data Scientists; O’Reilly Media, Inc.: Newton, MA, USA, 2018.

19. García, S.; Ramírez-Gallego, S.; Luengo, J.; Benítez, J.M.; Herrera, F. Big data preprocessing: Methods and prospects. Big Data Anal. 2016, 1, 9. [CrossRef]

20. Zhou, K.; He, W.; Xu, Y.; Xiong, G.; Cai, J. Feature selection and transfer learning for Alzheimer's disease clinical diagnosis. Appl. Sci. 2018, 8, 1372. [CrossRef]

21. Beheshti, I.; Demirel, H.; Matsuda, H.; Alzheimer's Disease Neuroimaging Initiative. Classification of Alzheimer's disease and prediction of mild cognitive impairment-to-Alzheimer's conversion from structural magnetic resource imaging using feature ranking and a genetic algorithm. Comput. Biol. Med. 2017, 83, 109-119. [CrossRef]

22. Ijaz, M.F.; Attique, M.; Son, Y. Data-Driven Cervical Cancer Prediction Model with Outlier Detection and Over-Sampling Methods. Sensors 2020, 20, 2809. [CrossRef] [PubMed]

23. Ali, F.; El-Sappagh, S.; Islam, S.R.; Ali, A.; Attique, M.; Imran, M.; Kwak, K.S. An intelligent healthcare monitoring framework using wearable sensors and social networking data. Future Gener. Comput. Syst. 2020, 114, 23-43. [CrossRef]

24. Khoshgoftaar, T.; Dittman, D.; Wald, R.; Fazelpour, A. First order statistics based feature selection: A diverse and powerful family of feature seleciton techniques. In Proceedings of the 2012 11th International Conference on Machine Learning and Applications (ICMLA 2012), Boca Raton, FL, USA, 12-15 December 2012; Volume 2, pp. 151-157.

25. Welling, M. Fisher Linear Discriminant Analysis | | University of Toronto. Technical Note. 2005. Available online: https: //www.cs.huji.ac.il/ \{\}csip/Fisher-LDA.pdf (accessed on 23 April 2019).

26. Lama, R.K.; Gwak, J.; Park, J.S.; Lee, S.W. Diagnosis of Alzheimer's disease based on structural MRI images using a regularized extreme learning machine and PCA features. J. Healthc. Eng. 2017, 1, 1-11. [CrossRef] [PubMed]

27. Abdi, H.; Williams, L.J. Principal Component Analysis. Wiley Interdiscip. Rev. Comput. Stat. 2010, 2, 433-459. [CrossRef]

28. Glozman, T.; Le, R.K. Classification of Alzheimer's Disease Based on White Matter Architecture. 2014. Available online: http:/ / cs229.stanford.edu/proj2014/Tanya\%20Glozman,\%20Rosemary\%20Le, \%20Classification\%20of\%20Alzheimer\%27s\% 20Disease\%20Based\%20on\%20White\%20Matter\%20Attributes.pdf (accessed on 23 April 2019).

29. Le, N.Q.K.; Do, D.T.; Hung, T.N.K.; Lam, L.H.T.; Huynh, T.T.; Nguyen, N.T.K. A computational framework based on ensemble deep neural networks for essential genes identification. Int. J. Mol. Sci. 2020, 21, 9070. [CrossRef]

30. Moradi, E.; Pepe, A.; Gaser, C.; Huttunen, H.; Tohka, J.; Alzheimer's Disease Neuroimaging Initiative. Machine learning framework for early MRI-based Alzheimer's conversion prediction in MCI subjects. Neuroimage 2015, 104, 398-412. [CrossRef]

31. Breiman, L. Random forests. Mach. Learn. 2001, 45, 5-32. [CrossRef] 
32. Grassi, M.; Rouleaux, N.; Caldirola, D.; Loewenstein, D.; Schruers, K.; Perna, G.; Dumontier, M. A Novel Ensemble-Based Machine Learning Algorithm to Predict the Conversion from Mild Cognitive Impairment to Alzheimer's Disease Using Socio-demographic Characteristics, Clinical Information and Neuropsychological Measures. Front. Neurol. 2019, 10, 756. [CrossRef]

33. Basaia, S.; Agosta, F.; Wagner, L.; Canu, E.; Magnani, G.; Santangelo, R.; Filippi, M.; Alzheimer's Disease Neuroimaging Initiative. Automated classification of Alzheimer's disease and mild cognitive impairment using a single MRI and deep neural networks. Neuroimage Clin. 2019, 21, 101645. [CrossRef]

34. Stamate, D.; Smith, R.; Tsygancov, R.; Vorobev, R.; Langham, J.; Stahl, D.; Reeves, D. Applying Deep Learning to Predicting Dementia and Mild Cognitive Impairment. In Proceedings of the AIAI International Conference on Artificial Intelligence Applications and Innovations, Halkidiki, Greece, 5-7 June 2020; Springer: Cham, Switzerland, 2020; pp. 308-319.

35. AnalyzeDirect. Available online: https://analyzedirect.com/analyze14/ (accessed on 15 December 2020).

36. FreeSurfer. Available online: https://surfer.nmr.mgh.harvard.edu/ (accessed on 15 December 2020).

37. Ruppert, G.C.; Teverovskiy, L.; Yu, C.P.; Falcao, A.X.; Liu, Y. A new symmetry-based method for mid-sagittal plane extraction in neuroimages. In Proceedings of the 2011 IEEE International Symposium on Biomedical Imaging: From Nano to Macro (ISBI 2011), Chicago, IL, USA, 30 March-2 April 2011; pp. 285-288.

38. Liu, Y.; Collins, R.T.; Rothfus, W.E. Robust midsagittal plane extraction from normal and pathological 3-D neuroradiology images. IEEE Trans. Med. Imaging 2001, 20, 175-192.

39. Teverovskiy, L.; Li, Y. Truly 3D midsagittal plane extraction for robust neuroimage registration. In Proceedings of the 3rd IEEE International Symposium on Biomedical Imaging: From Nano to Macro, Arlington, VA, USA, 6-9 April 2006; pp. 860-863.

40. Michalak, H.; Okarma, K. Improvement of image binarization methods using image preprocessing with local entropy filtering for alphanumerical character recognition purposes. Entropy 2019, 21, 562. [CrossRef] [PubMed]

41. Di Ruberto, C.; Fodde, G. Evaluation of Statistical Features for Medical Image Retrieval. In Proceedings of the International Conference on Image Analysis and Processing_ICIAP 2013, Naples, Italy, 9-13 September 2013; Springer: Berlin/Heidelberg, Germany, 2013; pp. 552-561.

42. Wang, Z.; Bovik, A.C.; Sheikh, H.R.; Simoncelli, E.P. Image quality assessment: From error measurement to structural similarity. IEEE Trans. Image Process. 2004, 13, 600-612. [CrossRef] [PubMed]

43. Kumar, V.; Gupta, P. Importance of statistical measures in digital image processing. Int. J. Emerg. Technol. Adv. Eng. 2012, 2, 56-62.

44. Esmael, B.; Arnaout, A.; Fruhwirth, R.K.; Thonhauser, G. A statistical feature-based approach for operations recognition in drilling time series. Int. J. Comput. Inf. Syst. Ind. Manag. Appl. 2015, 5, 454-461.

45. Yang, X.; Tridandapani, S.; Beitler, J.J.; Yu, D.S.; Yoshida, E.J.; Curran, W.J.; Liu, T. Ultrasound GLCM texture analysis of radiation-induced parotid-gland injury in head-and-neck cancer radiotherapy: An in vivo study of late toxicity. Med. Phys. 2012, 39, 5732-5739. [CrossRef]

46. Lee, C.; Zhang, A.; Yu, B.; Park, S. Comparison study between RMS and edge detection image processing algorithms for a pulsed laser UWPI (Ultrasonic wave propagation imaging)-based NDT technique. Sensors 2017, 17, 1224. [CrossRef]

47. Malik, F.; Baharudin, B. The statistical quantized histogram texture features analysis for image retrieval based on median and laplacian filters in the dct domain. Int. Arab J. Inf. Technol. 2013, 10, 1-9.

48. Ho, A.D.; Yu, C.C. Descriptive statistics for modern test score distributions: Skewness, kurtosis, discreteness, and ceiling effects. Educ. Psychol. Meas. 2015, 75, 365-388. [CrossRef]

49. Kalaiselvi, T.; Nagaraja, P. An automatic segmentation of brain tumor from MRI scans through wavelet transformations. Int. J. Image Graph. Signal Process. 2016, 8, 59.

50. Usman, K.; Rajpoot, K. Brain tumor classification from multi-modality MRI using wavelets and machine learning. Pattern Anal. Appl. 2017, 20, 871-881. [CrossRef]

51. O'Hara, S.; Draper, B.A. Introduction to the bag of features paradigm for image classification and retrieval. arXiv 2011, arXiv:1101.3354.

52. Rueda, A.; Arevalo, J.; Cruz, A.; Romero, E.; González, F.A. Bag of features for automatic classification of Alzheimer's disease in magnetic resonance images. In Proceedings of the CIARP 2012 17th Iberoamerican Congress on Pattern Recognition, Buenos Aires, Argentina, 3-6 September 2012; Springer: Berlin/Heidelberg, Germany, 2012; pp. 559-566.

53. Le, X.; Gonzalez, R. Pattern-based corner detection algorithm. In Proceedings of the 2009 6th International Symposium on Image and Signal Processing and Analysis, Salzburg, Austria, 16-18 September 2009; pp. 238-243.

54. Bay, H.; Ess, A.; Tuytelaars, T.; Van Gool, L. Speeded-up robust features (SURF). Comput. Vis. Image Underst. 2008, 110, 346-359. [CrossRef]

55. Bair, E. Semi-supervised clustering methods. Wiley Interdiscip. Rev. Comput. Stat. 2013, 5, 349-361. [CrossRef]

56. Lindholm, A.; Wahlström, N.; Lindsten, F.; Schön, T.B. Supervised Machine Learning. Lecture Notes for the Statistical Machine Learning Course. Available online: https://mwns.co/blog/wp-content/uploads/2020/01/Supervised-Machine-Learning.pdf (accessed on 15 December 2020).

57. Ghojogh, B.; Crowley, M. Linear and quadratic discriminant analysis: Tutorial. arXiv 2019, arXiv:1906.02590.

58. Evgeniou, T.; Pontil, M. Support vector machines: Theory and applications. In Machine Learning and Its Applications, Proceedings of the ACAI 1999 Advanced Course on Artificial Intelligence; Springer: Berlin/Heidelberg, Germany, 1999; pp. 249-257.

59. Jakkula, V. Tutorial on Support Vector Machine (SVM); School of EECS, Washington State University: Washington, DC, USA, 2006.

60. Zeidat, N.; Eick, C.F.; Zhao, Z. Supervised Clustering: Algorithms and Applications; University of Houston: Houston, TX, USA, 2005. 
61. Alom, M.Z.; Taha, T.M.; Yakopcic, C.; Westberg, S.; Sidike, P.; Nasrin, M.S.; Van Esesn, B.C.; Awwal, A.A.S.; Asari, V.K. The history began from alexnet: A comprehensive survey on deep learning approaches. arXiv 2018, arXiv:1803.01164.

62. Krizhevsky, A.; Sutskever, I.; Hinton, G.E. Imagenet classification with deep convolutional neural networks. Commun. ACM 2017, 60, 84-90. [CrossRef]

63. Yang, S.; Berdine, G. The receiver operating characteristic (ROC) curve. Southwest Respir. Crit. Care Chron. 2017, 5, 34-36. [CrossRef] 